\title{
Synthesis and structures of some new thiazolidin-4-ones and thiazolin-4-ones of anticipated biological activity
}

\author{
Kamal A. Kandeel* \\ Department of Chemistry, Faculty of Science, Ain Shams University, Abbassia 11566, \\ Cairo, Egypt \\ E-mail: kamalkandeel@hotmail.com
}

\begin{abstract}
The condensation of $\omega$-(4-formylphenoxy)acetophenone (3a) and its 4-bromo derivative (3b) with 2-thioxo-1,3-thiazolidin-4-one (4a) and 1,3-thiazolidin-2,4-dione (4b) in refluxing acetic acid in the presence of sodium acetate gave new E-5-arylmethylene-2-thioxo-1,3-thiazolidin-4one (5a and $5 \mathbf{b}$ ) and E-1,3-thiazolidin-2,4-dione (5c and $5 \mathbf{d}$ ) derivatives in good yields, respectively. However, treatment of $E$-(5a and $\mathbf{5 b}$ ) with piperidine (or morpholine) in refluxing ethanol afforded new E-5-arylmethylene-2-piperidinyl (or morpholinyl)-1,3-thiazolin-4-one derivatives (6a-d). The structures of all new compounds were established from microanalytical and spectral data.
\end{abstract}

Keywords: 2-Thioxo-1,3-thiazolidin-4-one derivatives, 1,3-thiazolidin-2,4-dione derivatives, 1,3-thiazolin-4-one derivatives

\section{Introduction}

Thiazolidin-4-ones and thiazolin-4-ones have been reported to demonstrate a wide range of pharmacological activities, which include antibacterial [1,2], antifungal [2], antimicrobial [3-5], antiviral [6], and anticonvulsant [7] effects. In continuation of our previous studies [8-11] on thiazolidin-4-ones and thiazolin-4-ones, we report here the synthesis of some new thiazolidin-4one (5) and thiazolin-4-one (6) derivatives of anticipated biological activity.

\section{Results and Discussion}

The aldehydes $\mathbf{3 a}$ and $\mathbf{3 b}$ were prepared by etherfication of $\omega$-bromoacetophenone (1a) and its 4bromo derivative (1b) with 4-hydroxybenzaldehyde (2) in refluxing dry acetone in the presence of anhydrous $\mathrm{K}_{2} \mathrm{CO}_{3}$. 


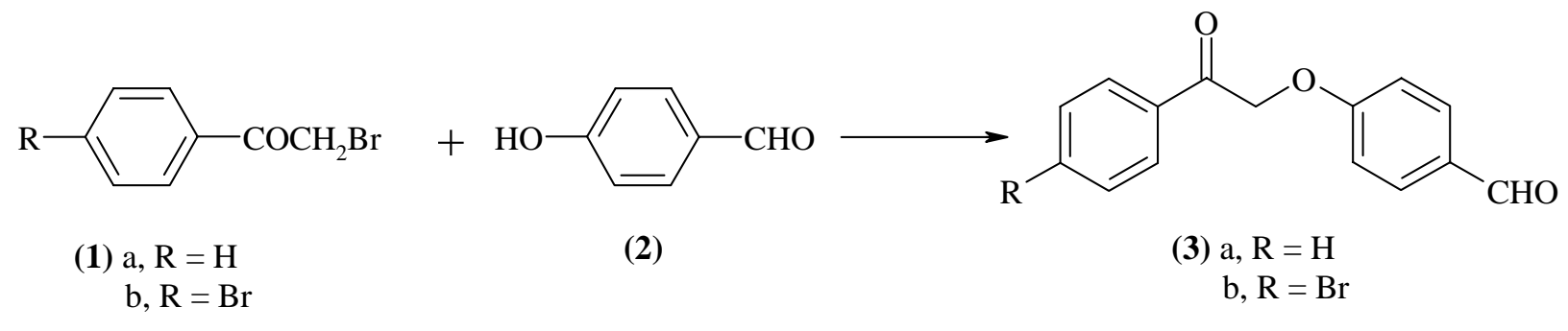

The aldehydes $\mathbf{3 a}$ and $\mathbf{3 b}$ were allowed to condense with 2-thioxo-1,3-thiazolidin-4-one (4a) and 1,3-thiazolidin-2,4-dione (4b) in refluxing glacial acetic acid in the presence of sodium acetate for $6 \mathrm{~h}$ to afford E-5-arylmethylene-2-thioxo-1,3-thiazolidin-4-one (5a and 5b) and E-5arylmethylene-1,3-thiazolidin-2,4-dione (5c and $\mathbf{5 d}$ ) derivatives, respectively, in good yields.

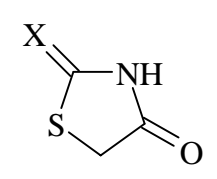

(4) a, $X=S$

b, $\mathrm{X}=\mathrm{O}$

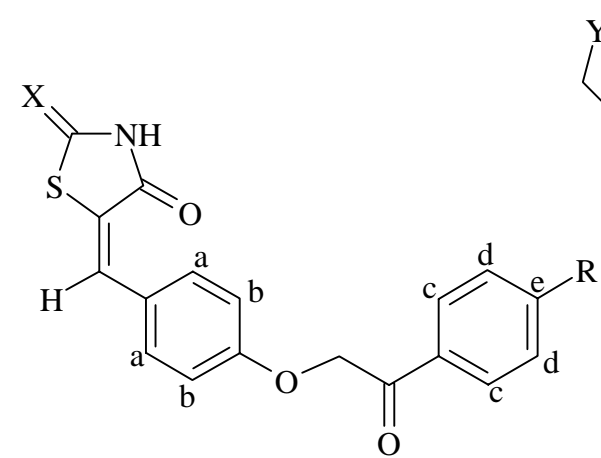

E-(5) a, $\mathrm{X}=\mathrm{S}, \mathrm{R}=\mathrm{H}$

$\mathrm{b}, \mathrm{X}=\mathrm{S}, \mathrm{R}=\mathrm{Br}$

c, $\mathrm{X}=\mathrm{O}, \mathrm{R}=\mathrm{H}$

$\mathrm{d}, \mathrm{X}=\mathrm{O}, \mathrm{R}=\mathrm{Br}$

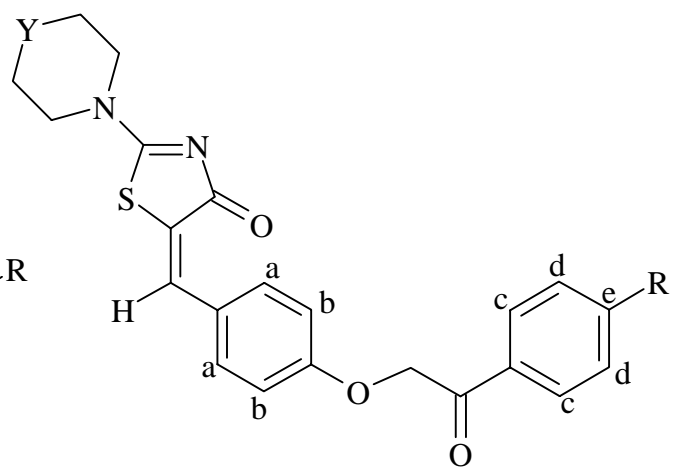

E-(6) a, $\mathrm{Y}=\mathrm{CH}_{2}, \mathrm{R}=\mathrm{H}$

b, $\mathrm{Y}=\mathrm{CH}_{2}, \mathrm{R}=\mathrm{Br}$

c, $\mathrm{Y}=\mathrm{O}, \quad \mathrm{R}=\mathrm{H}$

$\mathrm{d}, \mathrm{Y}=\mathrm{O}, \quad \mathrm{R}=\mathrm{Br}$

The structures of compounds $E$-(5a-d) were substantiated from microanalytical and spectral data. Thus, the infrared spectra showed absorptions characteristic for $\mathrm{NH}, \mathrm{C}=\mathrm{O}$ and $\mathrm{C}=\mathrm{S}$ groups in addition to other absorptions correlated to the assigned structures. Further, the ${ }^{1} \mathrm{H}$ NMR spectra showed signals corresponding to methylene, olefinic, aromatic and NH protons. Also, the EI-MS showed correct molecular ion peaks beside some of the abundant fragments. In addition, evidence to support the structural assignment was gained from the ${ }^{13} \mathrm{C}$ NMR spectrum of compound $5 \mathbf{a}$.

The reaction of compounds $E$-(5a and $\mathbf{5 b})$ with piperidine and morpholine in refluxing ethanol gave $E$-5-arylmethylene-2-piperidin-1-yl-1,3-thiazolin-4-one $E$-(6a and $\mathbf{6 b})$ and $E$-5arylmethylene-2-morpholin-4-yl-1,3-thiazolin-4-one $E$-(6c and $\mathbf{6 d )}$ derivatives, respectively, in very good yields.

The structures of compounds $E$-(6a-d) were elucidated from micro-analytical and spectral data. Thus, the infrared and ${ }^{1} \mathrm{H}$ NMR spectra showed absorptions correlated with the assigned structures. Moreover, the EI-MS showed correct molecular ion peaks in addition to some of the abundant peaks. 
The formation of compounds $E$-(6a-d) can be explained on the basis of a nucleophilic attack of piperidine or morpholine upon the $\mathrm{C}=\mathrm{S}$ group followed by elimination of hydrogen sulfide.

The E-configuration was assigned to structures $\mathbf{5}$ and $\mathbf{6}$ on the basis that a previous study [8] from this laboratory had identified the $E$ - and Z-isomers of analogous 5-arylmethylene compounds. It was shown that, the olefinic proton of the Z-configured isomers were more deshielded by the 4-oxo group of the thiazole moiety as compared with the E-counterparts and appeared at lower field ( $\delta \approx 8.00-8.20 \mathrm{ppm}$ ) relative to the $E$-isomer ( $\delta \approx 7.50-7.80 \mathrm{ppm})$.

\section{Experimental Section}

General Procedures. Melting points are uncorrected. IR spectra were measured on a Unicam SP 1200 spectrometer as $\mathrm{KBr}$ discs. Unless otherwise stated, the ${ }^{1} \mathrm{H}$ and ${ }^{13} \mathrm{C} \mathrm{NMR}$ spectra were measured in DMSO- $d_{6}$ on a Varian Gemini instrument at 200 and $50 \mathrm{MHz}$, respectively; in both cases, chemical shifts are given in ppm down-field from internal TMS. Mass spectra were recorded on a Shimadzu GC-MS Qp 1000 EX instrument operating at $70 \mathrm{eV}$.

Etherfication of $\omega$-bromoacetophenone (1a) and its 4-bromo derivative (1b) with 4hydroxybenzaldehyde (2). $\omega$-Bromoacetophenone (1a) or its 4-bromo derivative (1b) (10 mmol) was added to a mixture of 4-hydroxybenzaldehyde (2) (1.22 g, $10 \mathrm{mmol}$ ) and anhydrous $\mathrm{K}_{2} \mathrm{CO}_{3}(2.90 \mathrm{~g}, 30 \mathrm{mmol})$ in dry acetone $(50 \mathrm{ml})$. The mixture was refluxed on a water bath for 5-6 h, poured into iced cold water and then extracted with ether. The ether layer was dried by anhydrous $\mathrm{Na}_{2} \mathrm{SO}_{4}$ and rotatory evaporated to give $\mathbf{3 a}$ and $\mathbf{3 b}$.

$\omega$-(4-Formylphenoxy)acetophenone (3a). Brown viscous oil, 2.16 g (90\% yield. IR $\left(\mathrm{CHCl}_{3}\right)$ : $\bar{v}=3057\left(\mathrm{CH}_{\text {arom }}\right), 2940,2913$ and $2850\left(\mathrm{CH}_{\text {aliph }}\right), 1695(\mathrm{C}=\mathrm{O}), 1589$ and $1515(\mathrm{C}=\mathrm{C}), 820\left(\delta_{2-}\right.$ н), 750 and $690 \mathrm{~cm}^{-1}\left(\delta_{5-\mathrm{H}}\right)$.

$\omega$-(4-Formylphenoxy)-4-bromoacetophenone (3b). Brown viscous oil, $2.93 \mathrm{~g}$ (92\% yield). IR $\left(\mathrm{CHCl}_{3}\right): \bar{v}=3069\left(\mathrm{CH}_{\text {arom}}\right), 2958,2928$ and $2869\left(\mathrm{CH}_{\text {aliph }}\right), 1699(\mathrm{C}=\mathrm{O}), 1598$ and 1510 $(\mathrm{C}=\mathrm{C}), 826 \mathrm{~cm}^{-1}\left(\delta_{2-\mathrm{H}}\right)$

Condensation of aldehydes $3 a$ and $3 b$ with 2-thioxo-1,3-thiazolidin-4-one (4a) and 1,3thiazolidin-2,4-dione (4b). To a solution of $\mathbf{4 a}$ or $\mathbf{4 b}(10 \mathrm{mmol})$ in glacial acetic acid (25 ml) and anhydrous sodium acetate $(2.10 \mathrm{~g}, 30 \mathrm{mmol})$ was added aldehyde $\mathbf{3 a}$ or $\mathbf{3 b}$ (10 mmol). The reaction mixture was refluxed for $6 \mathrm{~h}$, cooled and then poured into iced cold water. The precipitated solid was filtered off and recrystalised from the proper solvent to give $E$-(5a-d).

E-5-[(4-Benzoylmethoxy)phenylmethylene]-2-thioxo-1,3-thiazolidin-4-one (5a). Pale yellow crystals, 2.30 g (65\% yield), m.p.: 235-237 $\mathrm{C}$ (acetic acid). IR: $\bar{v}=3240(\mathrm{NH}), 2920\left(\mathrm{CH}_{\text {arom}}\right)$, 2840 and $2740\left(\mathrm{CH}_{\mathrm{aliph}}\right), 1710(\mathrm{sh})$ and $1690(\mathrm{C}=\mathrm{O}), 1585(\mathrm{C}=\mathrm{C}), 1205(\mathrm{~S}-\mathrm{CS}-\mathrm{N}), 830\left(\delta_{2-\mathrm{H}}\right)$, 760 and $680 \mathrm{~cm}^{-1}\left(\delta_{5-\mathrm{H}}\right) .{ }^{1} \mathrm{H}$ NMR (DMSO-d, $\left.500 \mathrm{MHz}\right): \delta 5.39\left(\mathrm{~s}, \mathrm{CH}_{2}\right), 7.15$ (dd, 2, $\mathrm{H}_{\mathrm{b}}, J=7.4$ and $1.2 \mathrm{~Hz}$ ), 7.55 (dd, 2, $\mathrm{H}_{\mathrm{a}}, J=7.4$ and $1.2 \mathrm{~Hz}$ ), 7.58 (apparent t, 2, $\mathrm{H}_{\mathrm{d}}, J=8.0 \mathrm{~Hz}$ ), 7.59 (s, $=\mathrm{CH}$ ), 7.71 (apparent t, 1, $\mathrm{H}_{\mathrm{e}}, J=8.0 \mathrm{~Hz}$ ), 8.09 (dd, 2, $\mathrm{H}_{\mathrm{c}}, J=7.6$ and $1.2 \mathrm{~Hz}$ ), 13.75 (brs, NH, 
exchangeable with $\left.\mathrm{D}_{2} \mathrm{O}\right)$. EI-MS m/e (\%): $357\left(\mathrm{M}^{+}+2,10\right), 356\left(\mathrm{M}^{+}+1,36\right), 355\left(\mathrm{M}^{+}, 39\right)$, 270 (5), 269 (23), 268 ( $\mathrm{M}^{+}$- HNCS \& CO, 19), 149 (9), 106 (8), $105\left(\mathrm{C}_{6} \mathrm{H}_{5} \mathrm{CO}^{+}\right.$, base), 89 (12), 77 (35). ${ }^{13} \mathrm{C}$ NMR (DMSO- $\left.d_{6}, 125 \mathrm{MHz}\right): \delta 71.8\left(\mathrm{CH}_{2}\right), 117.3,127.4,129.3,130.4,133.0,134.0$, 135.4 and 135.7 (sp ${ }^{2}$ carbons), $161.5(\mathrm{C}=\mathrm{O}), 195.5(\mathrm{C}=\mathrm{S})$. Anal. Calcd. for $\mathrm{C}_{18} \mathrm{H}_{13} \mathrm{NO}_{3} \mathrm{~S}_{2}$ (355.430) requires : C, 60.82; H, 3.68; N, 3.94; S, 18.04\%. Found: C, 60.61, H, 3.57; N, 3.68; S, $17.89 \%$.

E-5-[4-(4-Bromobenzoylmethoxy)phenylmethylene]-2-thioxo-1,3-thiazo-lidin-4-one (5b). Pale yellow crystals, 3.0 g (70\% yield), m.p.: $253-254^{\circ} \mathrm{C}$ (ethanol). IR: $\bar{v}=3175$ (NH), 3085 ( $\mathrm{CH}_{\text {arom }}$ ), 2918 and $2850\left(\mathrm{CH}_{\text {aliph }}\right), 1706(\mathrm{C}=\mathrm{O}), 1587(\mathrm{C}=\mathrm{C}), 1210(\mathrm{~S}-\mathrm{CS}-\mathrm{N}), 820 \mathrm{~cm}^{-1}\left(\delta_{2-\mathrm{H}}\right) .{ }^{1} \mathrm{H}$ NMR (DMSO-d $d_{6}$ ): $\delta 5.73$ (s, $\mathrm{CH}_{2}$ ), 7.02 (dd, 2, $\mathrm{H}_{\mathrm{b}}, J=7.6$ and $1.2 \mathrm{~Hz}$ ), 7.43 (dd, 2, $\mathrm{H}_{\mathrm{a}}, J=7.6$ and $1.2 \mathrm{~Hz}$ ), 7.50 (s, =CH), 7.61 (dd, 2, $\mathrm{H}_{\mathrm{d}}, J=7.6$ and $1.2 \mathrm{~Hz}$ ), 7.99 (dd, 2, $\mathrm{H}_{\mathrm{c}}, J=7.6$ and 1.2 $\mathrm{Hz}$ ), 13.20 (brs, $\mathrm{NH}$, exchangeable with $\left.\mathrm{D}_{2} \mathrm{O}\right)$. EI-MS m/e (\%): $435\left(\mathrm{M}^{+}+2,16\right), 433\left(\mathrm{M}^{+}, 18\right)$, 348 (18), 346 ( $\mathrm{M}^{+}$-HNCS \& CO, 16), 186 (10), 185 (72), 184 (14), 183 (4- $\mathrm{BrC}_{6} \mathrm{H}_{4} \mathrm{CO}^{+}$, base), 157 (17), 155 (13), 152 (16), 151 (96), 133 (11), 90 (11), 79 (11), 77 (31), 76 (28), 75 (22), 74 (13), 65 (22), 64 (44), 63 (43), 62 (15), 61 (14). Anal. Calcd. for $\mathrm{C}_{18} \mathrm{H}_{12} \mathrm{BrNO}_{3} \mathrm{~S}_{2}$ (434.322) requires: C, 49.77; H, 2.78; N, 3.22; S, 14.76. Found: C, 49.63; H, 2.75; N, 3.09; S, 14.58\%.

E-5-[(4-Benzoylmethoxy)phenylmethylene]-1,3-thiazolidin-2,4-dione (5c). pale yellow crystals, 2.44 g (72\% yield), m.p.: $212-214^{\circ} \mathrm{C}$ (ethanol). IR: $\bar{v}=3160(\mathrm{NH}), 3030\left(\mathrm{CH}_{\text {arom }}\right)$, 2910, 2840 and $2770\left(\mathrm{CH}_{\text {aliph }}\right)$, 1728, $1710(\mathrm{sh})$ and $1690(\mathrm{C}=\mathrm{O}), 1585(\mathrm{C}=\mathrm{C}), 830\left(\delta_{2-\mathrm{H}}\right), 755$ and $690 \mathrm{~cm}^{-1}\left(\delta_{5-\mathrm{H}}\right) .{ }^{1} \mathrm{H}$ NMR (DMSO- $\left.d_{6}\right): \delta 5.62\left(\mathrm{~s}, \mathrm{CH}_{2}\right), 7.17$ (dd, 2, $\mathrm{H}_{\mathrm{b}}, J=7.8$ and $1.2 \mathrm{~Hz}$ ), $7.55-7.76\left(\mathrm{~m}, 6,2 \mathrm{H}_{\mathrm{a}}, 2 \mathrm{H}_{\mathrm{d}}, \mathrm{H}_{\mathrm{e}}\right.$ and $\left.=\mathrm{CH}\right), 8.05\left(\mathrm{dd}, 2, \mathrm{H}_{\mathrm{c}}, J=8.0\right.$ and $\left.1.2 \mathrm{~Hz}\right), 13.06$ (brs, NH, exchangeable with $\left.\mathrm{D}_{2} \mathrm{O}\right)$. EI-MS m/e (\%): $341\left(\mathrm{M}^{+}+2\right.$, 5), $340\left(\mathrm{M}^{+}+1,24\right), 399\left(\mathrm{M}^{+}, 27\right), 269$ (10), 268 ( $\left.\mathrm{M}^{+}-\mathrm{HNCO} \& \mathrm{CO}, 16\right), 149$ (5), 106 (8), 105 ( $\mathrm{C}_{6} \mathrm{H}_{5} \mathrm{CO}^{+}$, base), 77 (32). Anal. Calcd. for $\mathrm{C}_{18} \mathrm{H}_{13} \mathrm{NO}_{4} \mathrm{~S}$ (339.370) requires: $\mathrm{C}, 63.70 ; \mathrm{H}, 3.86$; $\mathrm{N}, 4.12$; $\mathrm{S}, 9.44 \%$. Found: $\mathrm{C}, 63.58 ; \mathrm{H}$, 3.74; N, 4.03; S, 9.27\%.

E-5-[4-(4-(4-Bromobenzoylmethoxy)phenylmethylene]-1,3-thiazolidin-2,4-dione (5d). Pale yellow crystals, $3.13 \mathrm{~g}$ (75\% yield), m.p.: $220-222^{\circ} \mathrm{C}$ (benzene/ethanol). IR: $\bar{v}=3250(\mathrm{NH})$, $3037\left(\mathrm{CH}_{\text {arom }}\right), 2921$ and $2852\left(\mathrm{CH}_{\text {aliph }}\right), 1729(\mathrm{sh})$ and $1703(\mathrm{C}=\mathrm{O}), 1610(\mathrm{C}=\mathrm{C}), 815 \mathrm{~cm}^{-1}\left(\delta_{2-\mathrm{H}}\right)$. ${ }^{1} \mathrm{H}$ NMR (DMSO- $d_{6}$ ): 5.58 (s, $\mathrm{CH}_{2}$ ), 7.15 (dd, 2, $\mathrm{H}_{\mathrm{b}}, J=7.8$ and $1.2 \mathrm{~Hz}$ ), 7.54 (dd, 2, $\mathrm{H}_{\mathrm{a}}, J=7.8$ and $1.2 \mathrm{~Hz}$ ), $7.60(\mathrm{~s},=\mathrm{CH}), 7.70\left(\mathrm{dd}, 2, \mathrm{H}_{\mathrm{d}}, J=7.8\right.$ and $1.2 \mathrm{~Hz}$ ), $8.12\left(\mathrm{dd}, 2, \mathrm{H}_{\mathrm{c}}, J=7.8\right.$ and 1.2 $\mathrm{Hz}), 13.45$ (brs, NH, exchangeable with $\left.\mathrm{D}_{2} \mathrm{O}\right)$. EI-MS m/e (\%): $419\left(\mathrm{M}^{+}+2\right.$, 31), $418\left(\mathrm{M}^{+}+1\right.$, 13), $417\left(\mathrm{M}^{+}, 16\right), 348\left(\mathrm{M}^{+}+2\right.$ - HNCO \& CO, 16), $346\left(\mathrm{M}^{+}-\mathrm{HNCO} \& \mathrm{CO}, 24\right), 342$ (18), 221 (25), 200 (37), 198 (17), 186 (10), 185 (60), 183 (4- $\mathrm{BrC}_{6} \mathrm{H}_{4} \mathrm{CO}^{+}$, 63), 157 (50), 162 (11), 156 (14), 155 (35), 151 (19), 150 ( $\mathrm{C}_{6} \mathrm{H}_{6} \mathrm{OS}$, base), 135 (12), 134 (10), 121 (13), 115 (15), 89 (14), 76 (13), 75 (14), 69 (18), 60 (70). Anal. Calcd. for $\mathrm{C}_{18} \mathrm{H}_{12} \mathrm{BrNO}_{4} \mathrm{~S}$ (418.262) requires: C, 51.68; $\mathrm{H}$, 2.89; N, 3.34; S, 7.66\%. Found: C, 51.53; H, 2.81; N, 3.29; S, 7.48\%. 
Reaction of 2-thioxo-1,3-thiazolidin-4-one derivatives $E$-(5a and $5 b$ ) with piperidine and morpholine. Piperidine or morpholine (6 mmol) was added to a solution of $E$-(5a) or $E$-(5b) (5 $\mathrm{mmol})$ in ethanol $(30 \mathrm{ml})$. The mixture was refluxed on a water bath until evolution of hydrogen sulfide ceased (5-6 h), whereupon it was cooled and then concentrated to afford $E$-(6a-d).

E-5-[(4-Benzoylmethoxy)phenylmethylene]-2-piperidin-1-yl-1,3-thiazolin-4-one

(6a). Orange crystals, 1.66 (82\% yield), m.p.: $164-166^{\circ} \mathrm{C}$ (ethanol). IR: $\bar{v}=3070\left(\mathrm{CH}_{\text {arom}}\right), 2950$ and $2880\left(\mathrm{CH}_{\text {aliph }}\right), 1710$ and $1690(\mathrm{C}=\mathrm{O}), 1605(\mathrm{C}=\mathrm{N}), 1570(\mathrm{C}=\mathrm{C}), 830\left(\delta_{2-\mathrm{H}}\right), 760$ and $690 \mathrm{~cm}^{-1}$ $\left(\delta_{5-\mathrm{H}}\right) .{ }^{1} \mathrm{H}$ NMR (DMSO- $\left.d_{6}\right): \delta 1.66$ (brs, 6, $-\left(\mathrm{CH}_{2}\right)_{3}-$ ), 3.44 (brs, 2, N-CH${ }_{2}$ ), 3.82 (brs, 2, N$\mathrm{CH}_{2}$ ), 5.65 (s, 2, $\mathrm{CH}_{2}$ ), 7.06 (dd, 2, $\mathrm{H}_{\mathrm{b}}, J=9.0$ and $1.8 \mathrm{~Hz}$ ), 7.54 (dd, 2, $\mathrm{H}_{\mathrm{a}}, J=8.7$ and $1.8 \mathrm{~Hz}$ ), 7.56 (s, 1, =CH), 7.75 (dd, 2, $\mathrm{H}_{\mathrm{d}}, J=8.7$ and $1.8 \mathrm{~Hz}$ ), 7.89 (apparent t, 1, $\mathrm{H}_{\mathrm{e}}, J=8.7 \mathrm{~Hz}$ ), 7.92 (dd, 2, $\mathrm{H}_{\mathrm{c}}, J=8.7$ and $1.8 \mathrm{~Hz}$ ). EI-MS m/e (\%): $408\left(\mathrm{M}^{+}+\right.$2, 3), $406\left(\mathrm{M}^{+}\right.$, 59), $268\left(\mathrm{M}^{+}\right.$$\mathrm{C}_{7} \mathrm{H}_{10} \mathrm{~N}_{2} \mathrm{O}$, 54), 150 (13), $105\left(\mathrm{C}_{6} \mathrm{H}_{5} \mathrm{CO}^{+}\right.$, base), 77 (45). Anal. Calcd. for $\mathrm{C}_{23} \mathrm{H}_{22} \mathrm{~N}_{2} \mathrm{O}_{3} \mathrm{~S}$ (406.502) requires: C, 67.95; H, 5.45; N, 6.89; S, 7.88\%. Found: C, 67.63; H, 5.21; N, 6.58; S, $7.74 \%$.

E-5-[4-(4-Bromobenzoylmethoxy)phenylmethylene]-2-peperidin-1-yl-1,3-thiazolin-4-one

(6b). Orange crystals, $\approx 2.0$ g (85\% yield), m.p.: $178-180^{\circ} \mathrm{C}$ (ethanol). IR: $\bar{v}=3050\left(\mathrm{CH}_{\text {arom }}\right)$, 2945, 2850 and $2760\left(\mathrm{CH}_{\text {aliph }}\right), 1705$ and $2690(\mathrm{C}=\mathrm{O}), 1603(\mathrm{C}=\mathrm{N}), 1559(\mathrm{C}=\mathrm{C}), 825 \mathrm{~cm}^{-1}\left(\delta_{2-\mathrm{H}}\right)$. ${ }^{1} \mathrm{H}$ NMR (DMSO- $d_{6}$ ): $\delta 1.62$ (brs, 6, - $\left(\mathrm{CH}_{2}\right)_{3}-$ ), 3.43 (brs, 2, $\mathrm{N}-\mathrm{CH}_{2}$ ), 3.85 (brs, 2, N-CH$)_{2}$ ), 5.71 (s, 2, $\mathrm{CH}_{2}$ ), 7.10 (dd, 2, $\mathrm{H}_{\mathrm{b}}, J=8.2$ and $1.2 \mathrm{~Hz}$ ), 7.62 (dd, 2, $\mathrm{H}_{\mathrm{a}}, J=8.0$ and $1.2 \mathrm{~Hz}$ ), 7.73 (s, 1, =CH), 7.79 (dd, 2, $\mathrm{H}_{\mathrm{d}}, J=8.7$ and $1.6 \mathrm{~Hz}$ ), 8.38 (dd, 2, $\mathrm{H}_{\mathrm{c}}, J=8.4$ and $1.8 \mathrm{~Hz}$ ). EI-MS m/e (\%):486 ( $\mathrm{M}^{+}+$2, 25), $484\left(\mathrm{M}^{+}, 25\right)$,348 (41), 347 (47), $346\left(\mathrm{M}^{+} \cdot-\mathrm{C}_{7} \mathrm{H}_{10} \mathrm{~N}_{2} \mathrm{O}, 47\right), 289$ (12), 288 (41), 185 (29), 183 (4- $\mathrm{BrC}_{6} \mathrm{H}_{4} \mathrm{CO}^{+}$, 24), 151 (11), 150 ( $\mathrm{C}_{8} \mathrm{H}_{6} \mathrm{OS}$, base), 149 (28), 121 (11), 110(12), 109(15), 91(11), 76(11), 69(12), 55(11). Anal. Calcd. for $\mathrm{C}_{23} \mathrm{H}_{21} \mathrm{BrN}_{2} \mathrm{O}_{3} \mathrm{~S}$ (485.394) requires : C, 56.91; H, 4.36; N, 5.77; S, 6.60\%. Found: C, 56.68; H, 4.29; N, 5.53; S, 6.41\%.

E-5-[4-(Benzoylmethoxy)phenylmethylene]-2-morpholin-4-yl-1,3-thiazolin-4-one

(6c). Orange crystals, 1.69 g (83\% yield), m.p.: $191-193^{\circ} \mathrm{C}$ (ethanol). IR: $\bar{v}=3060\left(\mathrm{CH}_{\text {arom }}\right), 2940$, 2870 and $2750\left(\mathrm{CH}_{\text {aliph }}\right), 1710$ and $1695(\mathrm{C}=\mathrm{O}), 1605(\mathrm{C}=\mathrm{N}), 1560(\mathrm{C}=\mathrm{C}), 836\left(\delta_{2-\mathrm{H}}\right), 760$ and $695 \mathrm{~cm}^{-1}\left(\delta_{5-\mathrm{H}}\right) .{ }^{1} \mathrm{H}$ NMR (DMSO- $\left.d_{6}\right): \delta 3.50$ (brs, 2, N-CH 2 ), 3.72 (brs, 4, $\mathrm{OCH}_{2}$ and N-CH$)_{2}$ ), 3.82 (brs, 2, $\mathrm{OCH}_{2}$ ), 5.71 (s, 2, $\mathrm{CH}_{2}$ ), 7.12 (dd, 2, $\mathrm{H}_{\mathrm{b}}, J=8.7$ and $1.2 \mathrm{~Hz}$ ), 7.65 (dd, 2, $\mathrm{H}_{\mathrm{b}}, J=8.7$ and $1.2 \mathrm{~Hz}$ ), $7.73(\mathrm{~s}, 1,=\mathrm{CH}), 7.93\left(\mathrm{dd}, 2, \mathrm{H}_{\mathrm{d}}, J=8.7\right.$ and 1.2 Hz), 8.03-8.16 (m, 3, $\mathrm{H}_{\mathrm{c}}$ and $\mathrm{H}_{\mathrm{e}}$ ). EI-MS m/e (\%): $410\left(\mathrm{M}^{+}\right.$+ 2, 1), $408\left(\mathrm{M}^{+}\right.$, 34), $268\left(\mathrm{M}^{+} \cdot-\mathrm{C}_{6} \mathrm{H}_{8} \mathrm{~N}_{2} \mathrm{O}_{2}, 65\right), 150$ (18), 105 $\left(\mathrm{C}_{6} \mathrm{H}_{5} \mathrm{CO}^{+}\right.$, base), 77(60). Anal. Calcd. for $\mathrm{C}_{22} \mathrm{H}_{20} \mathrm{~N}_{2} \mathrm{O}_{4} \mathrm{~S}$ (408.458) requires : C, 64.69; $\mathrm{H}, 4.93$; N, 6.85; S, 7.84\%. Found: C, 64.43; H, 4.82; N,6.71; S, 7.59\%.

E-5-[4-(4-Bromobenzoylmethoxy)phenylmethylene]-2-morpholin-4-yl-1,3-thiazolin-4-one (6d). Orange crystals, $\approx 2.0$ g (84\% yield), m.p.: $220-223^{\circ} \mathrm{C}$ (ethanol). IR: $\bar{v}: 3063\left(\mathrm{CH}_{\text {arom}}\right)$, 2920 and $2853\left(\mathrm{CH}_{\text {aliph }}\right), 1698$ and $1687(\mathrm{C}=\mathrm{O}), 1598(\mathrm{C}=\mathrm{N}), 1553(\mathrm{C}=\mathrm{C}), 820 \mathrm{~cm}^{-1}\left(\delta_{2-\mathrm{H}}\right) .{ }^{1} \mathrm{H}$ NMR (DMSO-d $d_{6}$ ): $\delta 3.62$ (brs, 2, $\mathrm{NCH}_{2}$ ), 3.71 (brs, 4, $\mathrm{NCH}_{2}$ and $\mathrm{OCH}_{2}$ ), 3.83 (brs, 2, $\mathrm{OCH}_{2}$ ), 5.61 (s, 2, $\mathrm{CH}_{2}$ ), 7.11 (dd, 2, $\mathrm{H}_{\mathrm{b}}, J=9.0$ and $1.8 \mathrm{~Hz}$ ), 7.57 (dd, 2, $\mathrm{H}_{\mathrm{a}}, J=9.0$ and $1.8 \mathrm{~Hz}$ ), 7.61 (s, 1, =CH), 7.80 (dd. 2, $\mathrm{H}_{\mathrm{d}}, J=8.4$ and $1.8 \mathrm{~Hz}$ ), 7.95 (dd, 2, $\mathrm{H}_{\mathrm{c}}, J=8.4$ and $1.8 \mathrm{~Hz}$ ). EI-MS m/e (\%): $488\left(\mathrm{M}^{+}+2,20\right), 486\left(\mathrm{M}^{+}, 21\right), 349$ (18), 348 (55), 347 (14) $346\left(\mathrm{M}^{+} \cdot-\mathrm{C}_{6} \mathrm{H}_{8} \mathrm{~N}_{2} \mathrm{O}_{2}, 67\right)$, 
345 (34), 291 (11), 290 (31), 186 (15), 185 (14), 182 (19), 171 (15), 152 (10), 151 (12), 150

( $\mathrm{C}_{8} \mathrm{H}_{6} \mathrm{OS}$, base), 121 (15), 112 (16), 90 (10), 89 (15). Anal. Calcd. for $\mathrm{C}_{22} \mathrm{H}_{19} \mathrm{BrN}_{2} \mathrm{O}_{4} \mathrm{~S}$ (487.370) requires: C, 54.21; H, 3.92; N, 5.74; S, 6.57\%. Found: C, 54.05; H, 3.73; N, 5.51; S, 6.32\%.

\section{References}

1. Mishra, P.; Gajbhiye, A.; Jain, S.K. Orient. J. Chem. 1996, 12, 325; Chem. Abstr. 1997, 126, 277425g.

2. Abdel-Halim, A.M.; Abdel-Aziz, R.M.; El-Dein, H.S.; El-Kafrawy, A.F. Indian J. Heterocycl. Chem. 1994, 4, 45; Chem. Abstr. 1995, 122, 105795d.

3. Pachhamia, V.L.; Parikh, A.R. Acta Cienc. Indica Chem. 1991, 17, 67; Chem. Abstr. 1992, 117, 26399w.

4. Ashour, F.A.; Habib, N.S.; Soliman, R.; El-Taibbi, M. Bull. Fac. Pharm. (Cairo Univ.) 1993, 31, 381; Chem. Abstr. 1993, 123, 198651n.

5. Bapodra, A.H.; Bharmal, F.; Parekh, H. Indian J. Pharm. Science 2002, 64, 501.

6. Shukle, S.K.; Singh, S.P.; Awasthi, L.P.; Mukherjee, D.D. Indian J. Pharm. Science 1982, 44, 153; Chem. Abstr. 1983, 99, 22365u.

7. Captan, G.; Ulusoy, N.; Ergenc, N.; Ekinic, A.C.; Vidin, A. Farmaco 1996, 51, 729; Chem. Abstr. 1996, 126, 157436q.

8. Omar, M.O.; Youssef, A.S.A.; Kandeel, K.A.; Phosphorus, Sulfur and Silicon 2000, 162, 25.

9. Kandeel, K.A.; Youssef, A.S.A. Molecules 2001, 6, 510.

10. Kandeel, K.A.; Youssef, A.M.; El-Bestawy, H.M.; Omar, M.O. Monatsh. Chemie 2002, 123, 1211.

11. Kandeel, K.A.; Youssef, A.M.; El-Bestawy, H.M.; Omar, M.O. J. Chem. Research(S) 2003, 682; J.Chem. Research (M) 2003, 1129. 